\title{
Extracellular protease imaging for cell mass tracking of xenografted human malignant pleural mesothelioma
}

\author{
LANLAN ZHOU $^{1}$, WAFIK EL-DEIRY ${ }^{1}$, WENGE WANG ${ }^{2}$, MARY E. INGRAM ${ }^{3-5}$ and SHARYN I. KATZ ${ }^{3-5}$ \\ ${ }^{1}$ Department of Medicine (Hematology/Oncology), Laboratory of Translational Oncology and Experimental \\ Cancer Therapeutics, Penn State College of Medicine, Penn State Hershey Cancer Institute, Penn State Hershey \\ Medical Center, Hershey, PA; ${ }^{2}$ Department of Internal Medicine, Mount Vernon Hospital, Mount Vernon, NY; \\ ${ }^{3}$ Department of Radiology, ${ }^{4}$ Institute for the Translational Medicine and Therapeutics, ${ }^{5}$ Abramson Cancer \\ Center, University of Pennsylvania Perelman School of Medicine, Philadelphia, PA, USA
}

Received January 17, 2012; Accepted February 20, 2012

DOI: $10.3892 /$ or.2012.1888

\begin{abstract}
Malignant pleural mesothelioma (MPM) is locally aggressive and challenging to quantitate non-invasively in vivo, particularly in orthotopic models of disease. We describe imaging of extracellular protease activity, typically elevated in locally aggressive tumors, as a novel method for tracking MPM in vivo. Mice bearing human MPM subcutaneous flank xenografted tumors were imaged with ProSense 680, an optical imaging agent of extracellular cysteine protease activity. The relative contribution of extracellular cysteine proteases to the ProSense tumor signal was estimated using RT-PCR quantitation of cysteine protease RNA expression of the MPM cell lines and compared to ArrayExpress microarray RNA expression data from human MPM tumors. Feasibility of orthotopic intraperitoneal MPM cell mass tracking with fluorescence signal was evaluated using CellVue Marooncoated MSTO-211H and compared to bioluminescent signal using luciferase-transfected MSTO-211H cells. ProSense 680 yielded a robust tumor signal in MPM subcutaneous grafts, primarily resulting from MPM secretion of cathepsin L demonstrated not only by RT-PCR data on MPM cell lines but also by microarray expression data from resected human patient tumors. CellVue Maroon intraperitoneal tumor signal was robust and durable indicating feasibility of intraperitoneal cell mass tracking of orthotopically-xenografted MPM. Optical imaging of extracellular cysteine protease activity is useful for tracking MPM tumor cell mass in vivo. Intraperitoneal MPM cell mass tracking of fluorescently labeled tumor is feasible.
\end{abstract}

Correspondence to: Dr Sharyn I. Katz, Department of Radiology, Perelman School of Medicine, Hospital of the University of Pennsylvania, 1 Silverstein Building, 3400 Spruce Street, Philadelphia, PA 19104, USA

E-mail: sharyn.katz@uphs.upenn.edu

Key words: mesothelioma, optical imaging, cathepsin, xenograft, thoracic malignancy, ProSense, orthotopic

\section{Introduction}

Malignant pleural mesothelioma (MPM) is an extremely invasive malignancy with complex mass geometry and asymmetric, discontinuous growth. MPM easily transgresses anatomical boundaries, including the pleura, pericardium and bone, typically respected by tumors, indicating robust secretion of extracellular proteases required for such aggressive local invasion. This pattern of tumor growth poses challenges in mouse models, even when measuring subcutaneous grafted tumors, since the invasive component is often significantly underestimated by external caliper measurement. Non-invasive caliper measurement is made impossible in orthotopic MPM xenografts, implanted in the pleura and peritoneal spaces. This is important since, in recent years, orthotopic models of malignancy, that is tumors grown in the anatomically 'natural' site of growth, have been demonstrated to often yield more robust growth. This phenomenon is likely due to locally available growth stimuli, allowing for more 'patient-like' patterns of local and metastatic invasion (1-6). For MPM, recent orthotopic pleural and intraperitoneal models have been introduced (7) as they are both typical spontaneous sites of human malignant mesothelioma (8) and do demonstrate robust tumor growth. A recent study successfully addressed intraperitoneal and intrapleural tumor mass visualization using red-shifted fluorescent protein (RFP) transfected MSTO-211H tumor cells (9). However, further development of simple, durable non-invasive methods for tracking of intraperitoneal MPM cell mass is highly valuable for non-invasive in vivo evaluation of tumor response in orthotopic models.

In this study we evaluate the feasibility of MPM tumor imaging with ProSense 680, an optical imaging agent which releases fluorescent signal when cleaved by extracellular protease activity. The primary protease contributors to ProSense 680 signaling are members of the cathepsin family of cysteine proteases (Fig. 3). Cathepsins are typically expressed within the endo/lysosomal intracellular compartment although are re-routed into the extracellular space in the context of specific physiological and pathophysiological processes, such as inflammation malignant local invasion and metastasis. 
The shunting of the cysteine cathepsins from the endo/lysosomal to secretory systems and release into the extracellular matrix is thought to mediated by downregulation of the insulinlike growth factor receptor-2. In the extracellular matrix, many of these proteases are able to degrade the surrounding stroma, a necessary step for malignant local and metastatic invasion. In particular cysteine protease cathepsins B, L, S and aspartyl protease cathepsin $\mathrm{D}$, effect robust degradation of components of the extracellular matrix (ECM), including collagen, fibronectin, proteoglycans, elastin and basement membrane, including Type-IV-collagen and laminin (10). While their activity and regulation is complex and not well understood, certain cathepsins have been observed to be routinely highly expressed in malignancy including cathepsins B, C, L, S and $\mathrm{X} / \mathrm{Z}$ of which only cathepsin $\mathrm{L}$ is nearly exclusively overexpressed in the setting of malignancy. The overexpression of some of these cathepsins, such as cathepsins $\mathrm{L}$ and B, are also associated with poor prognosis, increased tumor invasiveness and elevated frequency of metastasis. As a result, inhibitors of the cathepsin protease family are under development as potential therapeutic agents.

We describe the first report of imaging human malignant pleural mesothelioma with a physiologic, in vivo, fluorescent optical imaging probe of extracellular cathepsin protease activity. We also demonstrate feasibility of intraperitoneal MPM cell mass tracking using a cell surface-labeled fluorescent signal, approximating the signal in the extracellular compartment.

\section{Materials and methods}

Cell lines and cell culture. The human CALU-6 cell line was utilized for in vitro, and newly purchased from American Type Culture Collection (ATCC, Manasas, VA). Cells were maintained in a humidified sterile environment with $5 \% \mathrm{CO}_{2}$, at $37^{\circ} \mathrm{C}$. RPMI-medium supplemented with $10 \%$ heat-inactivated fetal calf serum with $1 \%$ penicillin and streptomycin (CM) was utilized to culture cell lines. Passage of cell lines was performed at 1:5 dilution after detachment using sterile $0.5 \%$ trypsin-EDTA solution.

Cell line transfection. The MSTO-211H cell line was transfected with the firefly luciferase gene using a retroviral vector as described previously (11).

Protocol approval was obtained from the IACUC at the University of Pennsylvania for xenograft formation prior to the use of animal models. Immunocompromised nu/ nu 6-week-old female mice were injected subcutaneously in each flank with $10^{7}$ cells suspended in a $50 \%$ sterile, endotoxin-free Matrigel solution $/ 50 \%$ CellGro Ex Vivo Medium Solution or intraperitoneally suspended in a $50 \%$ CellGro Ex Vivo/PBS solution. During tumor induction, mice were maintained at the 5 Richards Animal Facility at the University of Pennsylvania. After therapy/imaging completion, mice were euthanized with ketamine i.p. followed by cervical dislocation.

Optical imaging. After obtaining a pre-injection optical imaging acquisition in the target spectrum, optical imaging agent ProSense 680 ( $2 \mathrm{nmol} / 150 \mu \mathrm{l}$ in $1 \mathrm{X}$ PBS), purchased from
VisEn Medical, Inc. (Bedford, MA) was injected by tail vein and a time course of imaging (20 min, $6 \mathrm{~h}, 24 \mathrm{~h}$ and $48 \mathrm{~h}$ ) was acquired on the Maestro (CRI, Woburn MA), with appropriate excitation and absorbance filters. Mice were anesthetized with IP ketamine prior to each imaging time point.

CellVue Maroon labeling of MSTO-211H per the specifications of the manufacturers (Molecular Targeting Technologies, Inc., West Chester, PA) prior to engraftment. Image acquisition of the CellVue Maroon signal was acquired in the deep red spectrum (excitation wavelength $\max =647 \mathrm{~nm}$ and emission wavelength $\max =667 \mathrm{~nm}$ ). Post-processing of optical images was performed on the commercially available CRI Maestro software (Woburn, MA). The acquired optical signal, obtained as an image cube, was unmixed in order to separate dye signal from background autofluorescence. After signal unmixing, tumor signal uptake was quantitated with the CRI software.

$R T-P C R$. Total RNA was extracted from cultured MPM cell lines using the RNeasy Mini Kit (Qiagen, Valencia, CA) following the manufacturer's instructions. Reverse transcription was performed with random hexamers and SuperScript II Reverse Transcriptase (Invitrogen, Carlsbad, CA). Quantitative real-time polymerase chain reaction (qPCR) analysis was performed in triplicate using an ABI StepOnePlus Real-Time PCR System (Applied Biosystems, Foster City, CA) and SYBR Green PCR master mix (Applied Biosystems). Primers used for amplification were obtained from published literature and are as follows: cathepsin B, 5'-AAATCAGGCGTATACAA GCATGA-3' (forward) and 5'-GCCCAGAATGCGGATGG-3' (reverse); cathepsin L, 5'-CGGCATGAATGAAGA AGGAT-3' (forward) and 5'-TGGGCTTACGGTTTTGAAAG-3' (reverse); cathepsin D, 5'-ATCCCGCTGCACAAGTTCACGTCCATC CGC-3' and 5'-CCACCAGCTTCTGCTGCATCAGGTTG TCGAAGACG-3'; cathepsin S,5'-ATGAAACGGCTGGTTTG TGT-3' and 5'-CTAGATTTCTGGGTAAGAGGGAAAG CTAGC-3'; cathepsin K, 5'-CAGTGAAGAGGTGGTTC AGA-3' (forward) and 5'-AGAGTCTTGGGGCTCTACCTT-3' (reverse); cathepsin G, 5'-CTCAATATAATCAGCGGACC-3' (forward) and 5'-CCAGCAGTTTGAAGCTTCTC-3' (reverse); Klk5 (kallikrein-related peptidase 5), 5'-GCAAGACCCC CCTGGATGTG-3' (forward), 5'-TCCCAGAGGGCACG GTGTTA-3' (reverse); uPA, 5'-TTGCTCACCACAACGAC ATT-3' (forward) and 5'-GGCAGGCAGATGGTCTGTAT-3' (reverse); CD10 (membrane metallo-endopeptidase), 5'-TGT GGCCAGATTGATTCGTC-3' (forward) and 5'-TTGTAGGT TCGGCTGAGGCT-3' (reverse); $\beta$-actin, 5'-AGAAAATCT GGCACCACACC-3' (forward) and 5'-GGGGTGTTGAAGG TCTCAAA-3' (reverse); TATA-binding protein, 5'-CAGGAGC CAAGAGTGAAGAAC-3' (forward) and 5'-AGGAAATA ACTCTGGCTCATAACTACT-3' (reverse); GP3DH, 5'-CAA AGTTGTCATGGATGACC-3' (forward) and 5'-CCATGG AGAAGGCTGGGG-3' (reverse).

Statistical analysis RT-PCR data. The RT-PCR quantitative data were analyzed using an Excel Spreadsheet (97-2003). The difference between the mean CT values for the GADPDH controls and probe sets for each microarray plate were performed and the $\log 2$ ratio calculated and termed the 'actual value'. Actual values were then plotted on a bar graph for interpretation. 
Pre-injection
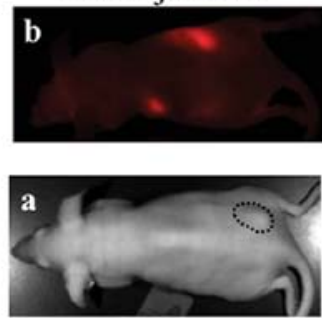

$20 \mathrm{~min}$
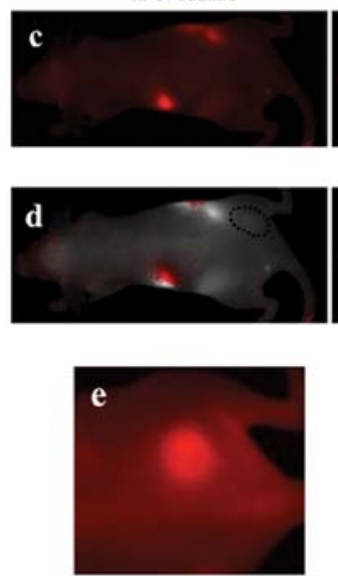

$24 \mathrm{~h}$
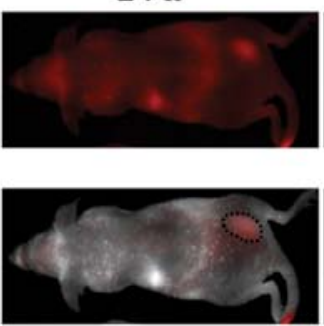

f

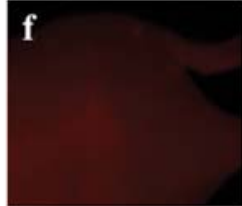

Figure 1. ProSense 680 optical probe enhancement of H2452 human malignant pleural mesothelioma subcutaneous right flank xenograft. The (a) white light image reveals a right flank subcutaneous xenograft human malignant pleural mesothelioma tumor (encircled with dotted line). Image acquisition using a deep red spectrum filter (b) prior to and (c) at $20 \mathrm{~min}, 24 \mathrm{~h}$ and $48 \mathrm{~h}$ following IV administration of ProSense 680 were then (d) unmixed from the background signal using CRI post-processing software and overlaid with the white light image for anatomic reference. Unmixed images demonstrate ProSense 680 accumulation in the right flank tumor indicating elevated intratumoral extracellular cysteine protease expression. Imaging of ProSense 680 signal (in a different mouse) using a deep red spectrum at (e) 3 weeks after injection with ProSense 680 reveals tumor retention of ProSense 680 signal which is (f) no longer visible with acquisition using the near infra-red spectrum, markedly reducing potential interference from this $680 \mathrm{~nm}$ wavelength signal in subsequent imaging.

a

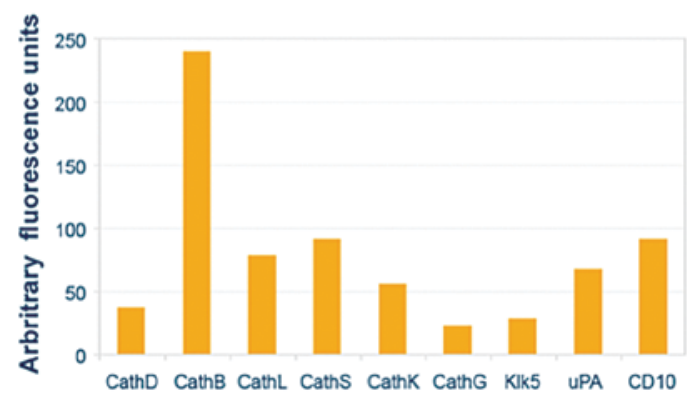

(reprinted with permission from ViseEn-Perkin Elmer)

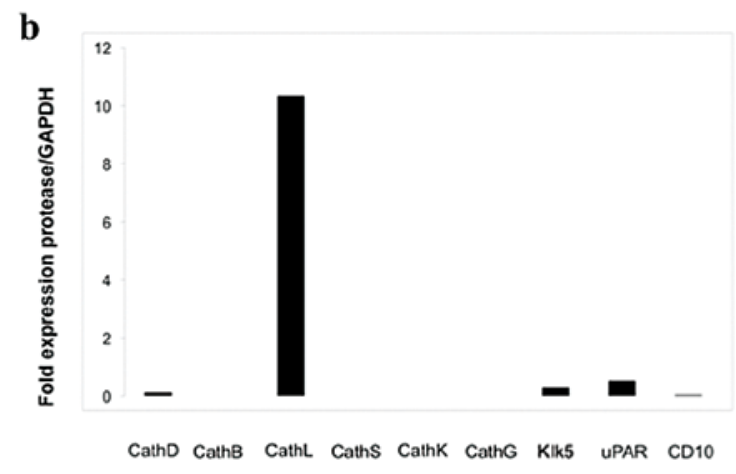

c

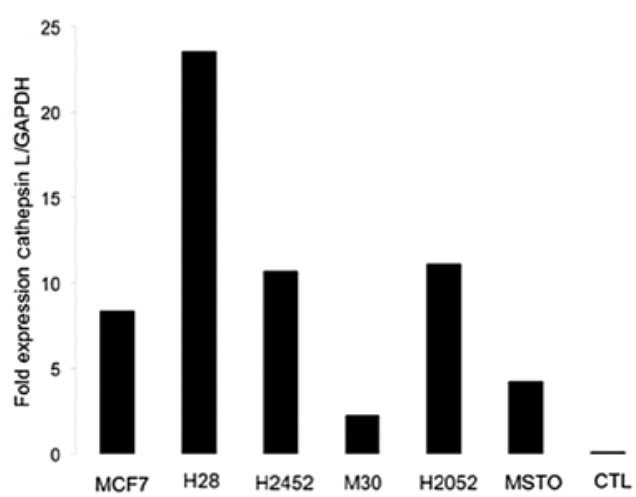

Figure 2. Gene expression profiling of human MPM cell lines demonstrates that xenograft MPM tumor ProSense 680 signal is derived primarily from cathepsin L. Data provided by the manufacturer of ProSense 680 (reprinted with permission from VisEn-Perkin Elmer) (a) demonstrates the relative fluorescence contributions of proteases contributing to the ProSense 680 signal. Average of expression data from all 5 MPM cell lines by RT-PCR (b) demonstrates that MPM xenograft ProSense 680 signal is predominately from cathepsin L. Comparison of relative levels of cathepsin L expression among the 5 tested MPM cell lines (c) reveals expression in all 5 lines, maximal in the $\mathrm{H} 28$ cell line.

Statistical analysis microarray data. RNA expression microarray data described previously (12) and deposited into the public database ArrayExpress (ID no. E-MTAB-47) was analyzed for expression of cysteine proteases. Fresh frozen tissue tissue samples were RNA extracted and run on the Affymetrix array HG-U133 Plus 2.0 microarray chip. 
a Mesothelioma vs. normal pleura protease expression b Pleural plaque vs. normal pleura protease expression
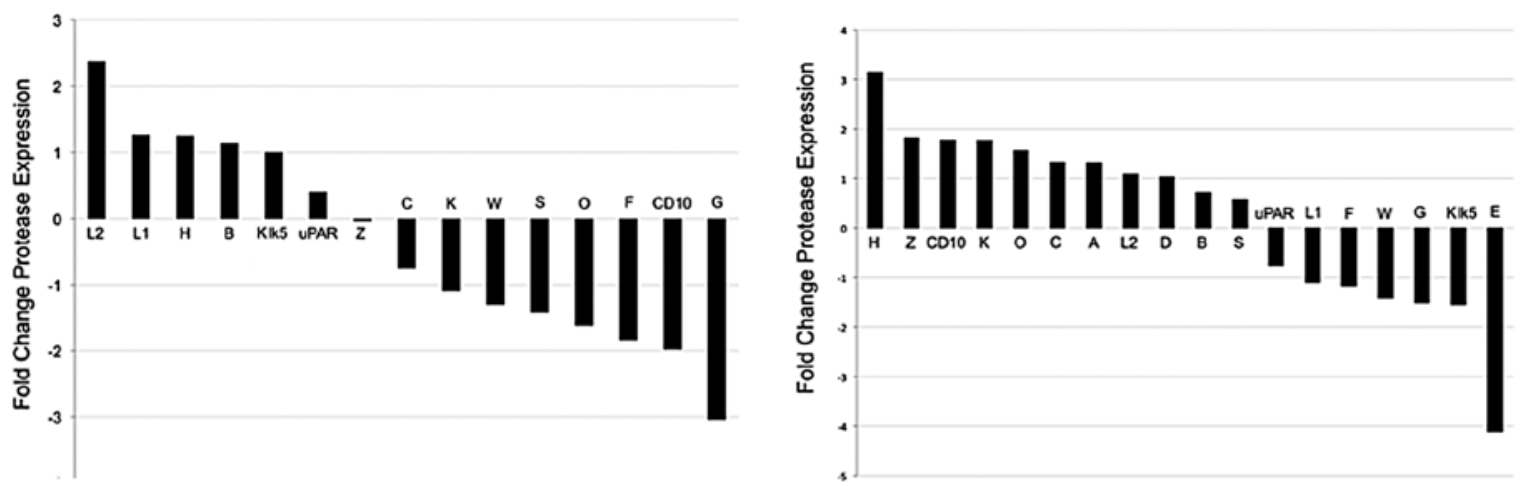

C Mesothelioma vs. pleural plaque protease expression

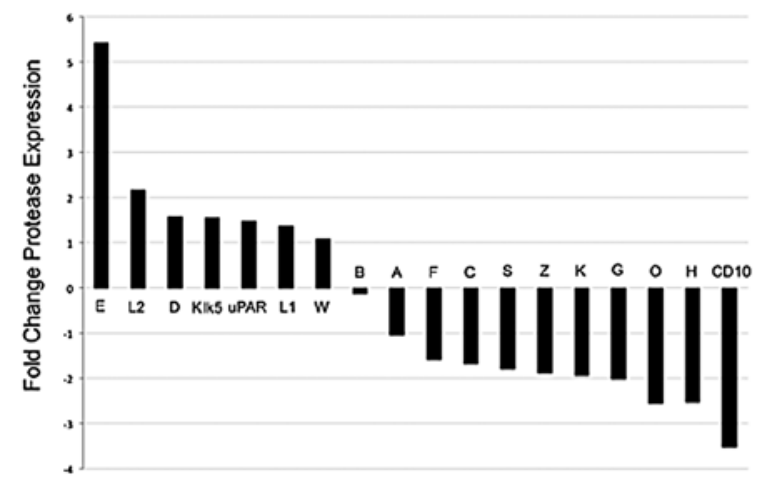

Figure 3. Gene expression profiling of human fresh frozen tissue also shows elevated expression of cathepsin L in MPM but not normal pleura or pleural plaque. RNA microarray expression data were downloaded from the ArrayExpress public data repository and analyzed for cathepsin expression. Elevated levels of cathepsin L, but not B or S, are observed in the MPM tumor relative to normal pleura (a) or pleural plaque (b). Analysis of pleural plaque relative to normal pleura (c) revealed a very different expression profile of cathepsins from MPM and consistent with inflammation.

A total of 12 human patient tissue samples were chosen from this publically available dataset, including 6 visceral pleura samples (A01.CEL, A04.CEL, A06.CEL, A08.CEL, A10. CEL, A11.CEL), 5 malignant pleural mesothelioma samples (A03.CEL, A12.CEL, A15.CEL, A16.CEL, A21.CEL) and 1 benign pleural plaque (A07.CEL), and Affymetrix.cel (probe intensity) files downloaded.

The patient samples were grouped into 3 categories, malignant pleural mesothelioma (5), normal pleura (6) and pleura plaque (1). The .cel files were imported into Partek Genomics Suite v6.5 (Partek Incorporated, St. Louis, MO) and GCRMA applied to normalize and summarize the data to the probeset level. We then applied a One-way ANOVA across the 3 groups and additionally calculated pairwise contrasts between each pair of groups. All calculated p-values were then corrected for False Discovery Rate using the method of Benjamini-Hochberg (13) as implemented in Partek. $\log 2$ ratio and fold-change was then calculated.

\section{Results}

MPM xenografts were imaged with the ProSense 680 optical imaging agent which yielded robust, durable tumor signal (Fig. 1a-d). This is a reflection of the constitutively elevated levels of extracellular proteases in malignant pleural mesothelioma. Once accumulated, ProSense 680 signal was still easily detectable within the xenografted tumors up to 3 weeks after injection (Fig. 1e) however, this signal is not visible in the near-infrared spectrum (Fig. 1f). This presents a limitation in that a limited range of agents can be administered coincidently or in tandem to allow the sufficient separation of the spectral emission curves vital for specific individual probe signal detection. The availability of a range of in vivo optical imaging agents in the 750-800 $\mathrm{nm}$ emission wavelengths allows for some flexibility in multispectral and longitudinal imaging with other molecular imaging agents.

ProSense 680 functions by integrating a quenched fluorophore-containing protease sensor in the cell surface lipid bilayer. Upon encountering an extracellular cathepsin protease, proteolysis ensues at multiple sites along its PEGylated polylysine backbone releasing fluorescence signal. While ProSense 680 activation can be induced by a range of proteases, including cathepsins $\mathrm{B}, \mathrm{D}, \mathrm{L}, \mathrm{S}, \mathrm{K}, \mathrm{G}$ and $\mathrm{Klk} 5$ and $\mathrm{uPA}$, the greatest activity of this agent is derived from interaction with cathepsins $\mathrm{B}$ and $\mathrm{L}$ and to a lesser extent cathepsin S (Fig. 2a). RNA expression analysis of the MPM cell lines used for xenograft formation was performed in order to determine the relative contributions of candidate extracellular proteases to ProSense signal in our xenograft mouse model of human MPM.

RT-PCR of RNA extracted from MPM cell lines, H28, H2052, M30, MSTO-211H and H2452 was performed using GAPDH as a control and revealed elevated levels of cathepsin $L$ relative 


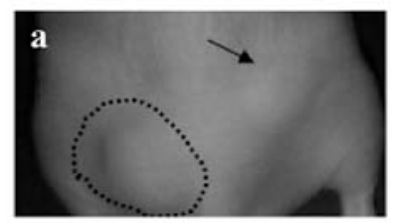

1 day

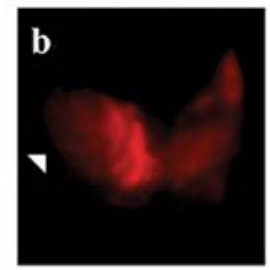

7 days

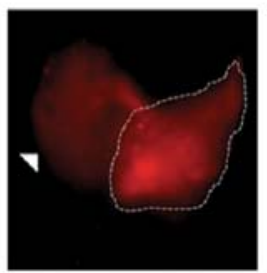

21 days

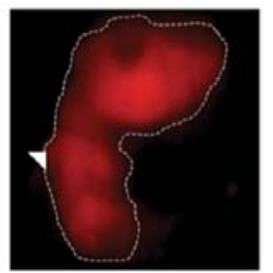

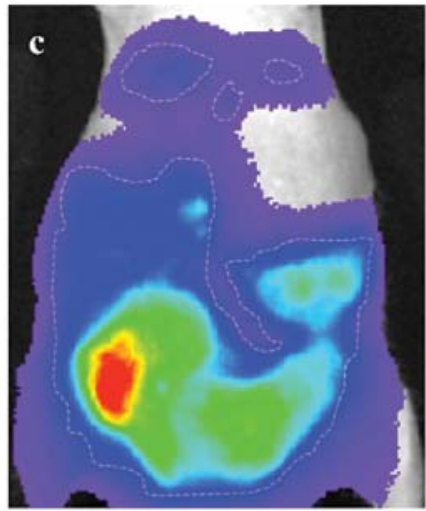

Figure 4. Subcutanous and orthotopic engraftment of MSTO-211H labelled with CellVue cell surface labeling agent. Human pleural mesothelioma cell line, MSTO-211H labelled with CellVue Maroon cell surface-labeled tumor implanted (a) subcutaneously in the left flank is faintly visible as a raised mass on the white light image (encircled with dashed line), on the left image, and deep red emission spectrum, on the right image, whereas the right flank unlabeled tumor (arrow) is only seen in the white light image since it contains no deep red fluorophore. MSTO-211H labelled with CellVue Maroon cell surface-labeled tumor implanted in the (b) intraperitoneal space and viewed in supine positioning $1 \mathrm{~d}, 7 \mathrm{~d}$ and $21 \mathrm{~d}$ following engraftment demonstrates the growth of tumor as initially layering fluorophore-labelled cell along the peritoneal covered bowel surfaces migrates and evolves into diffuse solid masses (encircled with dashed line). The arrowhead serves as a fixed point of reference, located at the lateral boundary of the right lower quadrant, to allow comparison between time points. For comparison, in a different mouse, (c) a heat map of luciferase signal emitted from luciferase expressing MSTO-211H cells imaged in supine positioning following intraperitoneal engraftment. The areas of highest signal (lighter colors) corresponds to area of greater tumor bulk whereas blues and purples colors depict areas of lower signal and therefore less tumor bulk.

to other tested extracellular proteases (Fig. 2b). Very low levels of UPAR (urokinase plasminogen activator surface receptor), $\mathrm{K} 1 \mathrm{k} 5$, CD10, cathepsin D, but no cathepsin B or S expression, was detected in the 5 malignant pleural mesothelioma cell lines. When cathepsin L expression was compared between the tested human MPM cell lines, the highest levels of expression was found in H28 and lowest in M30 (Fig. 2c). RT-PCR analysis of cathepsin family expression of the 5 human MPM cell lines used in this study demonstrated that the extracellular ProSense 680 signal observed within our in vivo MPM xenografted tumors is likely primarily a result of cathepsin L activity. Post-processing of publically available RNA expression microarray data on normal pleura, pleural plaque and malignant pleural mesothelioma was evaluated using a 2-fold change cut-off for overexpression/ underexpression. Data analysis demonstrated elevated levels of expression of cathepsin L, composed of relatively more L2 transcript than L1 transcript, in MPM relative to normal pleura (Fig. 3a) and relative to pleural plaque (Fig. 3b).

\section{Discussion}

Cathepsin L, is expressed preferentially in the setting of malignancy and inflammation. Overexpression of cathepsin $\mathrm{L}$ is seen almost exclusively in malignant cells, and is correlated with tumor aggressiveness manifested by local invasion and metastasis (14) thought to be a result of cathepsin L-mediated degradation of the extracellular matrix along with similar proteins such as other members of the cathepsin family and metalloproteinases. Interestingly, IL-6, a cytokine found in abundance in MPM pleural effusions (15), has been shown to increase the expression and secretion of cathepsin $\mathrm{L}(16,17)$. In our analysis of human RNA microarray expression data available on surgically obtained human tissues cathepsin L was elevated (greater than 2-fold increase) in MPM but not normal pleura or pleural plaque.

Other proteases that were potential ProSense 680 signal contributors did not reach 2-fold change including cathepsins D, B, S, uPAR, Klk5 and CD10. Cathepsin D, a lysosomal aspartic protease and potential contributor to ProSense signal, was slightly elevated in expression in MPM tissue samples (less than 2 -fold) relative to normal pleura and pleural plaque. However, while cathepsin D can contribute to ProSense 680 signal, it is proportionally a much weaker contributor than for cathepsin $\mathrm{L}$ (Fig. 2a). Other potential contributors to ProSense signal were either unchanged or underexpressed in human surgical tissues of MPM relative to pleural plaque or normal pleura. Cathepsin $\mathrm{H}$ and CD10, were underexpressed in MPM relative to pleural plaque (Fig. 3b) and cathepsin $\mathrm{H}$ was overexpressed in pleural plaque (Fig. 3c) relative to normal pleura. Cathepsin $\mathrm{H}$ is a serine protease responsible for overall degradation of lysosomal proteins and implicated in some malignancies.

Other cathepsins typically elevated in expression in the setting of inflammation, cathepsins H (18), C (18), K (19), B $(20,21)$ and $S(21)$, were also either decreased or little changed in expression in MPM tumor tissues relative to normal pleura and pleural plaque. Cathepsin $\mathrm{G}$, frequently expressed highly in inflammation, was decreased in both MPM (Fig. 3a) and pleural plaque (Fig. 3c) relative to normal pleura. The robust MPM tumor avidity for ProSense, and lack of expression of inflammatory cathepsins able to significantly contribute to ProSense signal, suggests extracellular protease imaging as a useful means of MPM imaging. 
In order to determine the feasibility of cell mass tracking using optical imaging agents in the intraperitoneal space, MSTO-211H human MPM tumor cells were coated with the CellVue Maroon, a non-specific cell surface labeling fluorophore that inserts non-specifically in the cell membrane prior to tumor xenografting. CellVue Maroon labeled MSTO-211H cells were then xenografted into nu/nu mice subcutaneously in the flank (Fig. 4a) and intraperitoneal (Fig. 4b) locations and tracked with serial optical image acquisition. Qualitatively, signal from MSTO-211H-labeled fluorescent probe CellVue Maroon was easily tracked for 3 weeks after grafting, although signal was not as robust as that generated from bioluminescence imaging. The dilution of CellVue Maroon signal, a result of cellular division and increasing cell mass, did not appear to impair qualitative tumor visualization. Following sacrifice, necropsy evaluation demonstrated correlation of tumor CellVue signal and cell mass location both in the subcutaneous and intraperitoneal locations after 3 weeks of growth. For comparison, imaging with bioluminescence signal (Fig. 4c) in the subcutaneous and intraperitoneal locations was performed using MSTO-211H transfected with the bioluminescent fluorophore, luciferase. Luciferase-transfected MSTO-211H demonstrated robust, tumor-specific signal that was easily tracked and appeared undiluted throughout the duration of the experiment.

CellVue, which coats the cell surface and thus approximates the extracellular space, demonstrated robust tumor cell mass fluorescent signal. The dilution of CellVue Maroon signal, a result of cellular division and increasing cell mass, did not appear to impair tumor visualization. While bioluminescent transfection mediated cellular imaging is generally known to have significant advantages over fluorescence, including robust signal, extremely low background and lack of signal dilution over time, fluorescent cell surface-labeling of cells has the advantage of ease of use while maintaining low background and durable signal. Caveats exist for both methods. For luciferase imaging, these caveats include the stability of cellular luciferase-transfection, imaging dependence on intravenous substrate injection and reports of potential biological impacts of luciferase upon tumor growth (22). For fluorescent-label cell surface coating, caveats include potential loss of label with concurrent IP delivery of drugs and interference with co-administered IV fluorescent physiological tracers. Nevertheless, cell surface coating of tumor cell approximates signal from the extracellular space.

In this study we demonstrated a novel method of imaging human MPM xenografts using an in vivo fluorescence imaging agent of extracellular protease activity, ProSense 680. We also demonstrated feasibility of cell mass tracking of MPM in the orthotopic intraperitoneal location using a fluorescent signal which address a significant caveat of intraperitoneal tumor engraftment since these tumors cannot be directly measured. Orthotopic modeling of human disease is often favored due to more physiologic, 'patient-like' disease patterning and often, as in the case of MPM, more robust tumor growth likely due to locally available tumor growth signals. Future directions include a quantitative study of fluorescently-labeled intraperitoneal cell mass, cell mass depth and measured signal intensity in order to define a non-invasive, method of quantitative intraperitoneal tumor mass measurement.

\section{Acknowledgements}

We would like to acknowledge Dr John Tobias, Interim Director, University of Pennsylvania Bioinformatics Core for his assistance in analysis of the microarray data.

\section{References}

1. Bibby MC: Orthotopic models of cancer for preclinical drug evaluation: advantages and disadvantages. Eur J Cancer 40: 852-857, 2004.

2. Grisanzio C, Seeley A, Chang M, et al: Orthotopic xenografts of RCC retain histological, immunophenotypic and genetic features of tumours in patients. J Pathol 225: 212-221, 2011.

3. Chan E, Patel A, Heston W and Larchian W: Mouse orthotopic models for bladder cancer research. BJU Int 104: 1286-1291, 2009.

4. Hoffman RM: Orthotopic metastatic mouse models for anticancer drug discovery and evaluation: a bridge to the clinic. Invest New Drugs 17: 343-359, 1999.

5. Yang M, Jiang P, Sun FX, et al: A fluorescent orthotopic bone metastasis model of human prostate cancer. Cancer Res 59: 781-786, 1999.

6. Gros SJ, Dohrmann T, Rawnaq T, et al: Orthotopic fluorescent peritoneal carcinomatosis model of esophageal cancer. Anticancer Res 30: 3933-3938, 2010.

7. Nakataki E, Yano S, Matsumori Y, et al: Novel orthotopic implantation model of human malignant pleural mesothelioma (EHMES-10 cells) highly expressing vascular endothelial growth factor and its receptor. Cancer Sci 97: 183-191, 2006.

8. Moolgavkar SH, Meza R and Turim J: Pleural and peritoneal mesotheliomas in SEER: age effects and temporal trends, 1973-2005. Cancer Causes Control 20: 935-944, 2009.

9. Yamaoka N, Kawasaki Y, Xu Y, et al: Establishment of in vivo fluorescence imaging in mouse models of malignant mesothelioma. Int J Oncol 37: 273-279, 2010.

10. Sloane BF: Cathepsin B and cystatins: evidence for a role in cancer progression. Semin Cancer Biol 1: 137-152, 1990.

11. Wang W and El-Deiry WS: Bioluminescent molecular imaging of endogenous and exogenous p53-mediated transcription in vitro and in vivo using an HCT116 human colon carcinoma xenograft model. Cancer Biol Ther 2: 196-202, 2003.

12. Roe OD, Anderssen E, Helge E, et al: Genome-wide profile of pleural mesothelioma versus parietal and visceral pleura: the emerging gene portrait of the mesothelioma phenotype. PLoS One 4: e6554, 2004.

13. Klipper-Aurbach Y, Wasserman M, Braunspiegel-Weintrob N, et al: Mathematical formulae for the prediction of the residual beta cell function during the first two years of disease in children and adolescents with insulin-dependent diabetes mellitus. Med Hypotheses 45: 486-490, 1995.

14. Lankelma JM, Voorend DM, Barwari T, et al: Cathepsin L, target in cancer treatment? Life Sci 86: 225-233, 2010.

15. DeLong P, Carroll RG, Henry AC, et al: Regulatory T cells and cytokines in malignant pleural effusions secondary to mesothelioma and carcinoma. Cancer Biol Ther 4: 342-346, 2005.

16. Yamaguchi T, Naruishi K, Arai H, Nishimura F and Takashiba S: IL-6/sIL-6R enhances cathepsin B and L production via caveolin-1-mediated JNK-AP-1 pathway in human gingival fibroblasts. J Cell Physiol 217: 423-432, 2008.

17. Gerber A, Wille A, Welte T, Ansorge S and Buhling F: Interleukin- 6 and transforming growth factor- $\beta 1$ control expression of cathepsins B and $L$ in human lung epithelial cells. J Interferon Cytokine Res 21: 11-19, 2001.

18. D'Angelo ME, Bird PI, Peters C, Reinheckel T, Trapani JA and Sutton VR: Cathepsin $\mathrm{H}$ is an additional convertase of progranzyme B. J Biol Chem 285: 20514-20519, 2010.

19. Asagiri M, Hirai T, Kunigami T, et al: Cathepsin K-dependent toll-like receptor 9 signaling revealed in experimental arthritis. Science 319: 624-627, 2008.

20. Nagai A, Murakawa Y, Terashima M, et al: Cystatin C and cathepsin B in CSF from patients with inflammatory neurologic diseases. Neurology 55: 1828-1832, 2000.

21. Martin SL, Moffitt KL, McDowell A, et al: Association of airway cathepsin $\mathrm{B}$ and $\mathrm{S}$ with inflammation in cystic fibrosis. Pediatr Pulmonol 45: 860-868, 2010.

22. Brutkiewicz S, Mendonca M, Stantz K, et al: The expression level of luciferase within tumour cells can alter tumour growth upon in vivo bioluminescence imaging. Luminescence 22: 221-228, 2007. 